\title{
Physiology-based model of cell viscoelasticity
}

\author{
José J. Muñoz and Santiago Albo ${ }^{\dagger}$ \\ Department of Applied Mathematics III, Laboratori de Càlcul Numèric (LaCàN) and \\ Universitat Politècnica de Catalunya (UPC), 08036 Barcelona, Spain
}

\begin{abstract}
The measured viscoelastic properties of biological tissues is the result of the passive and active response of the cells. We propose an evolution law of the remodeling process in the cytoskeleton which is able to mimic the viscous properties of biological cellular tissues. Our model is based on dynamical changes of the resting length. We show that under the small strain regime, the linear rheology models are recovered, with the relaxation time being replaced by the cell resistance to remodel. We implement the one-dimensional model into network systems of two and three dimensions, and show that the same conclusions may be drawn for those systems.
\end{abstract}

\section{INTRODUCTION}

It is well recognized that cell viscosity is not solely due to the fluid part of the cytoplasm (water) but also due to cell activity [1-4]. However, when retrieving characteristic viscous coefficients of cells, there is a wide spectrum of values that have been employed, which range from $\eta=4.210^{-3} \mathrm{~Pa} \mathrm{~s}$, according to the Brownian motion of molecules in embryonic cells of Drosophila melanogaster [5], to $\eta=10^{5} \mathrm{~Pa}$ s for cells at its wing imaginal disk, a value deduced from relaxation experiments [6,7]. While the former values are close to water viscosity $\left(\eta=8.910^{-4} \mathrm{~Pa} \mathrm{~s}\right)$, the latter coefficient is in fact similar to the viscosity of olive oil or ketchuplike materials. Hence, in order to shed light into the mechanisms that cause the cellular response of the cell, it seems necessary to bridge the measured viscosity and the cellular biomolecular processes. This need has been already recognized, for instance, in Refs. [8,9]. This work aims precisely to present a simple phenomenologically based model that relates the observed cell viscosity with the cytoskeleton activity and its protein content, which in fact may attain up to $40 \%$ of the cell volume [10].

The effects of F-actin disruption on the loss and storage modulus have been experimentally observed in Refs. [11,12]. On the other hand, important efforts on the development of models that couple motor proteins and the viscoelastic properties have been carried out. According to these models, the measured viscoelastic properties of cells are the combination of the passive cell network [13-15] and also a consequence of the active response of the motor proteins $[2,3,16]$. In the latter case, the cell activity is taken into account by adding active or motor stresses $[16,17]$, which will influence the cytoskeleton equilbrium. In particular, the works in Refs. [4,18] consider a Kelvin-Voigt model with additional myosin contractile stresses. Similarly, Kruse and Jülicher [8] also consider forces from motors at the filaments, which are mediated through diffusion equations.

The introduction of additional cellular stresses may be interpreted as a Kelvin-Voigt-type extension of the model. We here instead consider the ability of the actin filaments to change dynamically their resting length, which may be

\footnotetext{
*j.munoz@upc.edu

†santi.albo@gmail.com
}

interpreted as a Maxwell-type extension where the cell is able to change its reference configuration. We interpret such changes of the resting length as a by-product of either the (de-)polymerization in the cytoskeleton or the remodeling of the cytoskeleton cross-links.

In order to ease the description of our model in the next section, some well-known classical results for onedimensional viscoelasticity will be recast. After applying an oscillatory strain with the form $\varepsilon(t)=\varepsilon_{o} e^{i \omega t}$, the resulting stresses are in general expressed as

$$
\sigma(t)=\left(E^{\prime}+i E^{\prime \prime}\right) \varepsilon(t),
$$

with $E^{\prime}$ and $E^{\prime \prime}$ the storage and loss modulus, respectively, from which we can also define the phase angle or mechanical loss $\delta=\arctan \left(E^{\prime \prime} / E^{\prime}\right)$ [1,19]. For a Kelvin-Voigt and a Maxwell rheological model, depicted in Figs. 1(a) and 1(b), the values of $E^{\prime}, E^{\prime \prime}$, and $\tan \delta$ are given in Table I.

Clearly, biological tissues do not obey the simple discrete Maxwell and Kelvin-Voigt models but rather a combination of them [e.g., the standard linear solid in Fig. 1(c)] or, in fact, a rheological model with a continuous spectrum, which can be constructed by using Boltzmann linearity assumption of discrete responses. For a definition of the standard solid model and other common combinations of springs and dashpots, the reader is referred, for instance, to Ref. [19]. In the next section we will focus our attention to a one-dimensional model with a discrete spectrum and eventually comment in the discussion section the extension of these results to models with a continuous spectrum.

\section{MODEL DEFINITION AND SOLUTION}

Our physical picture is the following. When a set of cross-linked actin filaments in the cytoskeleton is subjected to a macroscopic strain, it stretches as a result of two combined phenomena: (i) a reversible (elastic) deformation and a (ii) nonreversible remodeling and lengthening. The latter is illustrated in Fig. 2 and phenomenologically explained as the remodeling of the cross-links and a (de-)polymerization process of the filaments. In addition, we hypothesize that (iii) the current resting length $L$ of the combined filaments, that is, the total length of the filaments when subjected to zero loads at their ends, is proportional to the elastic strain. The previous picture may be mathematically described in a simple manner 


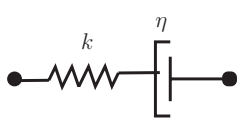

(a)

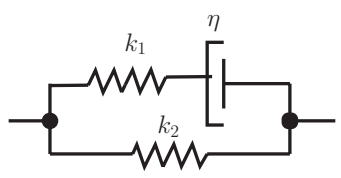

(c)

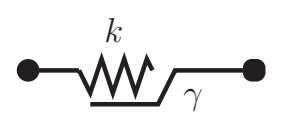

(d)
FIG. 1. Representation of (a) Kelvin-Voigt, (b) Maxwell, (c) standard solid models, and (d) proposed elastic element with changing resting length.

by assuming that the resting length satisfies the following evolution law:

$$
\frac{\dot{L}}{L}=\gamma \varepsilon^{e},
$$

that is, the relative changes of the resting length is proportional to the current elastic strain. The latter will be assumed as a linear measure of the deformation and, thus, will be defined as $\varepsilon^{e}=(l-L) / L$, with $l$ the current total length of the network. This strain measure differs from the apparent strain $\varepsilon=\left(l-L_{0}\right) / L_{0}$, with $L_{0}$ the initial length and resting length of the one-dimensional element, which, due to Eq. (1), will differ from $L$. The parameter $\gamma$ will be called the remodeling rate, which represents the resistance of the network to adapt its configuration to the new imposed deformation.

The relation in (1) is a simple linear law for the rate of remodeling, but without further experimental evidence, it seems as yet unnecessary to test more complicated relations. We note that the relation between actomyosin activation and the viscous properties of the tissue have been recently reported in Ref. [11], where an inhibition of the actomyosin cytoskeleton induces an increase of the viscous properties of the tissue. The main implications of the proposed law in Eq. (1) are that (i) no lengthening occurs if the filament is not subjected to stretch and that (ii) the filament tends to reduce the amount of elastic strain.

We will next apply the evolution law in (1) to a single one-dimensional element with initial length $L_{0}$ and prescribed displacement $u(X=0)=0$ and analyze the response when subjected to different boundary conditions at $X=L$. We will assume that the total deformation of the filament is solely due to the changes in the resting length and to a purely linear elastic deformation. For reasons that will be made clear in our

TABLE I. Values of storage modulus $E^{\prime}$, loss modulus $E^{\prime \prime}$, and tangent of phase angle for Kelvin-Voigt and Maxwell rheological models.

\begin{tabular}{lccc}
\hline \hline & $E^{\prime}$ & $E^{\prime \prime}$ & $\tan \delta$ \\
\hline Kelvin-Voigt & $k$ & $\eta \omega$ & $\frac{\eta}{k} \omega$ \\
Maxwell & $\frac{k \eta^{2} \omega^{2}}{k^{2}+\eta^{2} \omega^{2}}$ & $\frac{k^{2} \eta \omega}{k^{2}+\eta^{2} \omega^{2}}$ & $\frac{k}{\eta} \omega^{-1}$ \\
\hline \hline
\end{tabular}

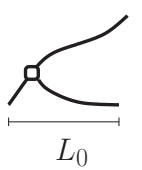

(a)

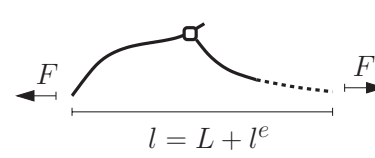

(b)

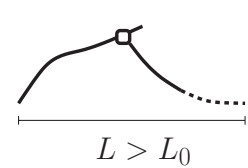

(c)
FIG. 2. Schematic of strain-induced changes in the resting length $L$ of a reduced system with two filaments and a cross-link (white circle). (a) Initial configuration with resting length equal to $L_{0}$. (b) Configuration under an applied load. (c) New unstrained configuration with modified resting length $L>L_{0}$. Dotted lines indicate extensions of the filament due to filament polymerization.

discussion, we will compare the response of this active model with a linear elastic Maxwell model.

\section{A. Constant stress (creep)}

We will next apply a constant stress $\sigma_{0}$ at the end $X=L_{0}$. Combining the evolution law in Eq. (1) with the equilibrium equation of a purely elastic element yields the following differential equation:

$$
\frac{\dot{L}}{L}=\frac{\sigma_{0}}{k} \gamma .
$$

After integrating this equation with the initial condition $L(t=0)=L_{0}$ we obtain the following expressions of the apparent strain and resting and total lengths:

$$
\begin{aligned}
& \varepsilon(t)=\left(\frac{\sigma_{0}}{k}+1\right) e^{\sigma_{0} \gamma t / k}-1, \quad L(t)=L_{0} e^{\sigma_{0} \gamma t / k}, \\
& l(t)=L_{0}\left(\frac{\sigma_{0}}{k}+1\right) e^{\sigma_{0} \gamma t / k} .
\end{aligned}
$$

Instead, in a linear Maxwell element, the governing equation reads

$$
\dot{\varepsilon}=\frac{\dot{\sigma}}{k}+\frac{\sigma}{\eta}
$$

where the total strain $\varepsilon$ is the sum of the elastic and viscous components, i.e., $\varepsilon=\varepsilon^{e}+\varepsilon^{v}$. The apparent strain and total length are given in this case by

$$
\begin{aligned}
& \varepsilon(t)=\frac{\sigma_{0}}{k}\left(1+\frac{t}{\tau}\right), \\
& l(t)=L_{0}\left[1+\frac{\sigma_{0}}{k}\left(1+\frac{t}{\tau}\right)\right],
\end{aligned}
$$

with $\tau=\eta / k$ the relaxation time of the Maxwell element. Although the apparent (total) strain differs in the two cases, the elastic strain $\varepsilon^{e}$, which is given by the constitutive law $\sigma=k \varepsilon^{e}$, is the same and equal to $\sigma_{0} / k$ for the active and Maxwell element. The inspection of Eqs. (3) and (2) reveals that close to the initial configuration $(t \rightarrow 0)$ and for small elastic strains $\left(\sigma_{0} / k \rightarrow 0\right)$, the apparent lengths are equal in the two elements if $\gamma$ is replaced by $\tau^{-1}$, as the plot of the evolution of $l$ in Fig. 3(a) confirms. Consequently, for a constant applied load, the active lengthening may be thought as a resistance to the stretching of the material. 


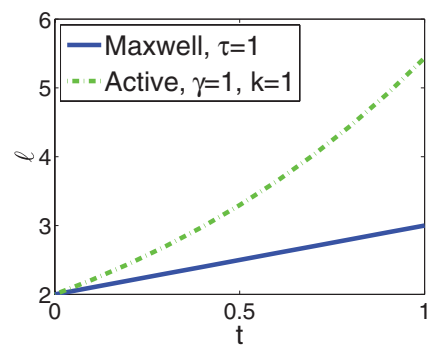

(a)

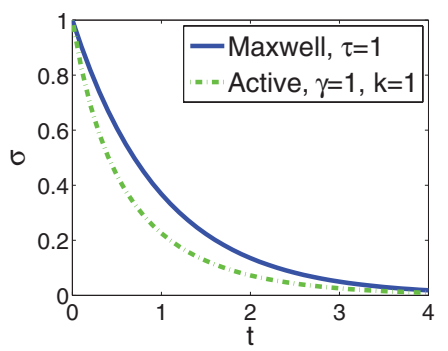

(b)
FIG. 3. (Color online) Comparison of the Maxwell and active model. (a) Evolution of the apparent length $l$ on a creep test (constants stress equal to $\sigma_{0}=1.0$ ). (b) Evolution of the stress $\sigma$ on a stress relaxation test (constant displacements and an initial strain equal to $\left.\varepsilon_{0}=1.0\right)$.

\section{B. Linear displacements}

We apply the linear monotonic boundary conditions $l\left(X=L_{0}\right)=L_{0}+c t, t>0$, with $c$ as an arbitrary constant. According to the evolution law in (1), the resting length $L$ and elastic strain $\varepsilon^{e}$ are given by

$$
\begin{aligned}
L & =L_{0}+c t+\frac{c}{\gamma}\left(e^{-\gamma t}-1\right), \\
\varepsilon^{e} & =\frac{1-e^{-\gamma t}}{\gamma L_{0} / c+\gamma t+e^{-\gamma t}-1},
\end{aligned}
$$

which are independent of the stiffness $k$. For a Maxwell model with relaxation time $\tau$, the elastic strain is instead given by

$$
\varepsilon^{e}=\frac{\sigma}{k}=\frac{\tau c}{L}\left(1-e^{-t / \tau}\right)
$$

Figure 4(a) shows the trend of the elastic strains for the Maxwell viscoelastic element, and for the active lengthening with $\gamma=1$ and $\gamma=0.25$, when using the parameters $c=1.0$ and $L_{0}=1.0$. While in a viscoelastic material the elastic strain and the stress approach a constant value, in the element with active lengthening they attain a maximum value and eventually asymptotically converge to zero, that is, the difference between the apparent length $l$ and the resting length $L$ is equal to $l-L=\gamma\left(e^{-\gamma t}-1\right) / c$, which approaches the value $c / \gamma$ as $t$ increases. However, due to the increase of the resting length $L$, the elastic strain converges towards 0 . As expected, the

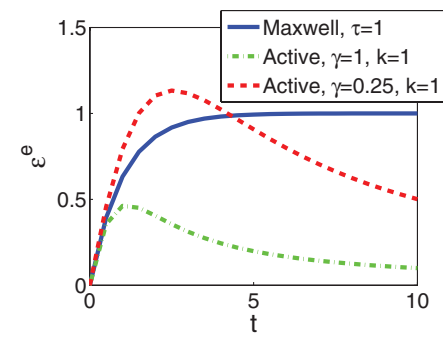

(a)

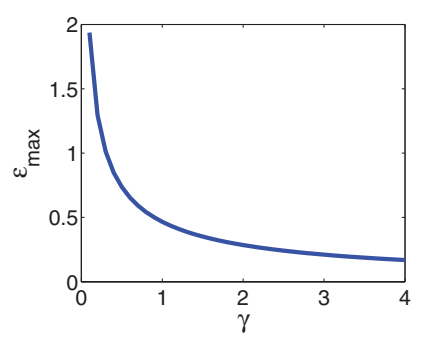

(b)
FIG. 4. (Color online) (a) Evolution of the elastic strains $\varepsilon^{e}$ when applying a linearly increasing displacement on a Maxwell model and on an active element with $\gamma=1$ and $\gamma=0.25$. (b) Relation between the maximum elastic strain $\varepsilon_{\max }^{e}$ and the remodeling rate $\gamma$. The curves were obtained using the parameters $c=1.0$ and $L_{0}=1.0$. smaller the remodeling rate, the slower the cell rearranges, and, consequently, the larger the maximum strain becomes. Figure 4(b) shows the maximum elastic strain $\varepsilon_{\max }^{e}$ as a function of the remodeling rate, which confirms this trend. The reduction on $\varepsilon_{\max }^{e}$ can be interpreted as a maximum yield stress, similarly to the model considered in Ref. [20], which in our case depends on the remodeling rate of the cell.

Note that the trend of $\varepsilon^{e}$ as $t \rightarrow \infty$ substantially differs for $\gamma>0$ and for $\gamma=0$, that is, when assuming a remodeling process or not. In the latter case, we have that $L=L_{0}$ and $\varepsilon^{e}=\frac{c}{L_{0}} t$, while, in the former case, $\varepsilon^{e}$ approaches the value 0 as $t \rightarrow \infty$. Consequently, according to the proposed model, the cell activity has effects not only on the apparent viscoelasticy but also on the maximum and asymptotic value of the stresses.

\section{Constant displacements (stress relaxation)}

We will analyze the stress relaxation process by, first, applying a monotonic displacement during a short period of time $T$ such that $l(T)=L_{1}$ and subsequently maintaining the apparent length of the element. From the result in Eq. (4), and using the fact that $L_{1}=L_{0}+c T$, we have that during the time period $t \in[0, T]$, the resting length is given by $L(T)=L_{1}+c / \gamma\left(e^{-\gamma T}-1\right)$. If we consider a sudden prescribed displacement $\left[T=\left(L_{1}-L_{0}\right) / c \rightarrow 0\right]$, we have that $L(T) \rightarrow L_{0}$, that is, the resting length remains unchanged after subjecting the element to a sudden prescribed displacement. The evolution of $L(t)$ after $t=T$ is then given by the solution of Eq. (1) with $l(t)=L_{1}$, which yields

$$
L(t)=L_{1}-\varepsilon_{0} L_{0} e^{-\gamma t}, \quad \sigma(t)=k \frac{\varepsilon_{0} e^{-\gamma t}}{1+\varepsilon_{0}\left(1-e^{-\gamma t}\right),}
$$

with $\varepsilon_{0}=\left(L_{1}-L_{0}\right) / L_{0}$. The comparison of the last expression with the stress evolution after applying the same experiment to a Maxwell element [19],

$$
\sigma(t)=\varepsilon_{0} k e^{-t / \tau}
$$

reveals that although the values of $\sigma(t)$ for $t=0$ and $t \rightarrow \infty$ are the same in both cases, their evolutions slightly differ. However, for the particular case of small strains $\left(\varepsilon_{0} \ll 1\right)$, the stress relaxation curves approach each other. The plot of the stress evolution in Fig. 3(b) shows the similarity of the two cases for $\varepsilon_{0}=1.0$.

\section{Oscillatory displacements and stresses}

In order to mimic standard rheology analysis of tissues with oscillatory boundary conditions at variable frequencies, we will analyze the behavior of the active element for such conditions. When the element is subjected to the displacement boundary condition $l(t)=L_{0}\left[1+\varepsilon_{0} \sin (\omega t)\right]$, the current active lengthening $L(t)$ is given by the solution of the differential equation in (1) as follows:

$$
L(t)=L_{0}+\frac{L_{0} \varepsilon_{0}}{\gamma^{2}+\omega^{2}}\left[\gamma \omega e^{-\gamma t}+\gamma^{2} \sin (\omega t)-\gamma \omega \cos (\omega t)\right] .
$$

It follows that the elastic strain is equal to

$$
\varepsilon^{e}(t)=\frac{\frac{\varepsilon_{0}}{\gamma^{2}+\omega^{2}}\left[\gamma \omega e^{-\gamma t}+\gamma^{2} \sin (\omega t)-\gamma \omega \cos (\omega t)\right]}{1+\frac{\varepsilon_{0}}{\gamma^{2}+\omega^{2}}\left[\gamma \omega e^{-\gamma t}+\gamma^{2} \sin (\omega t)-\gamma \omega \cos (\omega t)\right]} .
$$




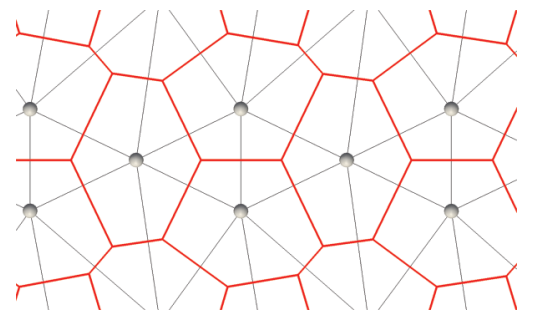

FIG. 5. (Color online) Scheme of the cell-centered model: Spheres represent cell nuclei, thin lines cell-cell contacts, and thick lines the cell boundaries. All forces between neighboring cells are represented by a single truss element, which is constructed by using a Delaunay trinagularization of the cell-nuclei. The cell boundary corresponds to the Voronoi diagram of the triangularization. (See, for instance, Ref. [21] for the definitions of Delaunay triangularization and the Voronoi diagram.)

By assuming small strains, and a linear elastic relationship $\sigma=k \varepsilon^{e}$, the steady-state response of the stresses at $t \rightarrow \infty$ has the following expression:

$$
\sigma(t) \stackrel{t \rightarrow \infty}{=} k \varepsilon_{0} \frac{\omega^{2} \sin (\omega t)+\gamma \omega \cos (\omega t)}{\gamma^{2}+\omega^{2}+\varepsilon_{0} \gamma^{2} \sin (\omega t)-\varepsilon_{0} \gamma \omega \cos (\omega t)},
$$

Due to the small strains assumption $\left(\left|\varepsilon^{e}\right| \ll 1\right)$, we can then compute the dynamic moduli and phase angle $\delta$ as

$$
\begin{aligned}
E^{\prime} & =\frac{k \omega^{2}}{\omega^{2}+\gamma^{2}}, \quad E^{\prime \prime}=\frac{k \gamma \omega}{\omega^{2}+\gamma^{2}}, \\
\delta & =\arctan \left(\gamma \omega^{-1}\right) .
\end{aligned}
$$

It can be verified that if instead of oscillatory strains we apply the oscillatory stresses $\sigma(t)=\sigma_{0} \sin (\omega t)$, the equilibrium condition $k \varepsilon(t)^{e}=\sigma(t)$, together with the evolution law in (1) with $L(t=0)=L_{0}$, we obtain the same expressions of the dynamic moduli and the phase angle for $\left|\sigma_{0} / k\right| \ll 1$. By comparing the expressions in (5) with the relations in Table I, we have that, again, the active element behaves like the Maxwell element but replacing $\gamma$ with $k / \eta=\tau^{-1}$.

\section{ANALYSIS OF NETWORKS}

We now extend the one-dimensional model to a network system of trusses, where each node represents the cell nucleus, and each truss carries the intra- and intercellular forces between two adjacent cells. Figure 5 shows a portion of such a network, where the position of the nuclei is determined by nodal equilibrium. The figure also depicts the cell boundaries,

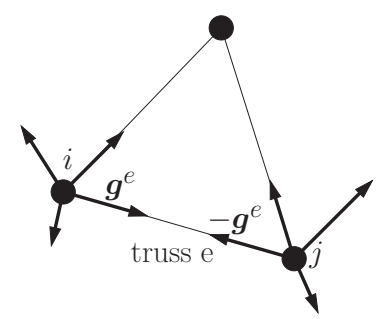

FIG. 6. Nodal forces due to the presence of truss $e$ between nodes $i$ and $j$.

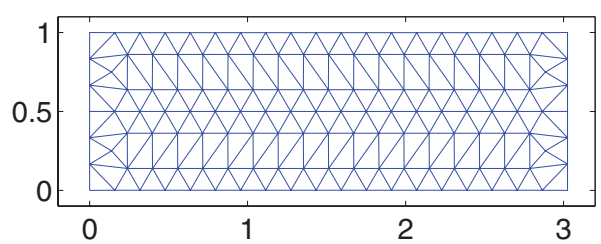

FIG. 7. (Color online) Two-dimensional mesh employed for the oscillatory boundary conditions, stress relaxation and linear monotonic displacements.

which correspond to the Voronoi diagram of the node-to-node connectivity, constructed by Delaunay triangularization [21].

We have implemented the one-dimensional model described in the previous section for each truss of the network, which can deform in any arbitrary direction and which can include arbitrary nonlinear viscoelastic laws for Kelvin-Voigt and Maxwell models. The positions of the cell nuclei are found by imposing that the sum of all the forces acting on each node $i$ must be equal to zero, i.e.,

$$
\sum_{e=1}^{N_{i}} \boldsymbol{g}^{e}=\mathbf{0}, \quad i=1, \ldots, N_{\text {nodes }},
$$

where $g^{e}$ is the force along truss $e$ and the sum is computed for all the $N_{i}$ elements $e$ connected to node $i$. Figure 6 illustrates the forces acting on a generic element $e$ between nodes $i$ and $j$. This force acts along the direction of the truss, but its magnitude is given by the rheological law described in the previous section, where $l$ is now the apparent distance between nodes $i$ and $j$. Further details of the implementation may be found in Ref. [22]. We will verify in this section whether the response analyzed so far for one truss is also inherited by the truss network.

It must be noted that the treatment of the cellular tissue as a truss network substantially differs from the continuum hypothesis. We have chosen the former approach for its simplicity in dealing with nonlinear variations of the resting length and with phenomenologically based remodeling process. Note that relating the stresses and the strains in the continuum requires the definition of the remodeling rate in a tensorial manner (different rates for different directions and deformation modes). We here circumvent these difficulties by constructing the network system, which in fact is also closer to the network

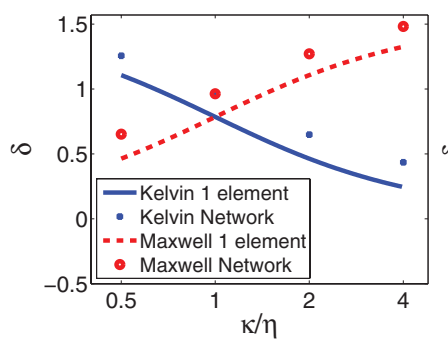

(a)

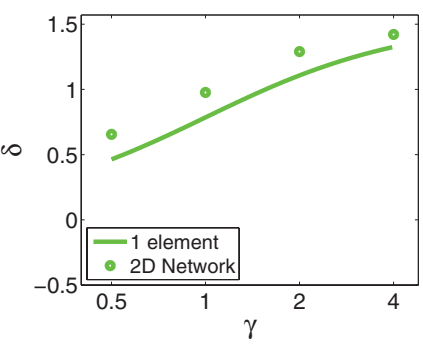

(b)
FIG. 8. (Color online) Comparison of the phase angle $\delta$ for the one-dimensional case (continuous lines) and the two-dimensional network (dots), computed from the analysis of the reactions when applying oscillatory displacements. (a) Kelvin-Voigt and Maxwell models. (b) Active element. 

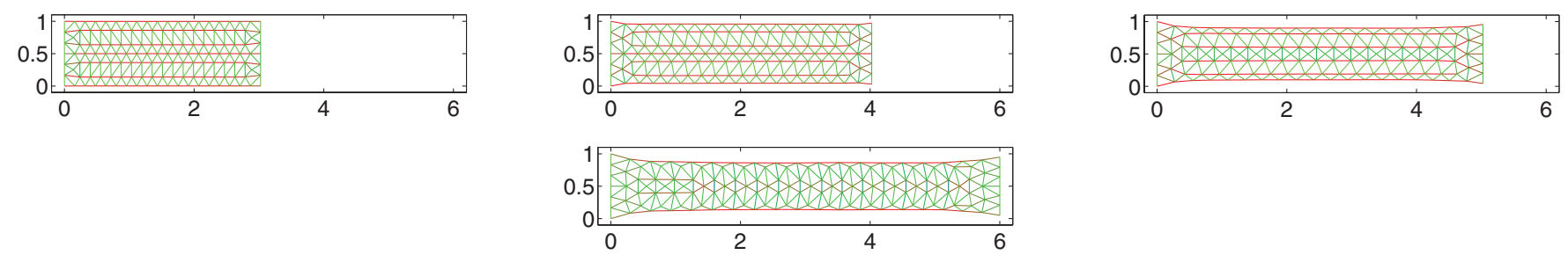

FIG. 9. (Color online) Resulting meshes for a network subjected to an increasing displacement $x=X_{0}+0.5 t$ and simultaneously to a Delaunay triangularization. Snapshots correspond to times $t=0, t=2, t=4$, and $t=6$.

structure of the cytoskeleton. We recognize that the network discretization of continua has some restrictions with respect to the continuum media [23]. Among them we highlight that (i) the material properties of a continuous material such as the Poisson's ratio depend on the topology of the truss network and that (ii) a continuous nodal stress field compatible with the tractions field will not exist in general or, in other words, a stress field $\sigma$ such that the truss tractions $g$ satisfy at each node $\boldsymbol{g}=\boldsymbol{\sigma} \boldsymbol{n}$, with $\boldsymbol{n}$ the direction of the truss, will not exist. This is due to the fact that our traction field is not derived from a continuous stress field but instead computed from nodal equilibrium. Despite these differences, we have tested that for the examples we show here, the actual network topology has minimal effects on the behavior of the remodeling process.

\section{A. Model analysis under oscillatory loads}

We have used the two-dimensional patch shown in Fig. 7. The left end has been fixed, while the right end has been subjected to oscillatory loads or displacements. The material properties are such that $\tau=1$.

In order to determine the phase angle for the patches, a Fourier analyses of the oscillatory response of the reactions and the imposed displacements at one of the ends was performed. The values of $\delta$ for different imposed frequencies are given in Fig. 8, which shows that the two-dimensional values are slightly greater than the corresponding one-dimensional results. This difference is presumably due to the contribution of the diagonal trusses, which have a different response than the horizontal trusses. In both cases, though, the trends of a single truss and the network patch are very similar.
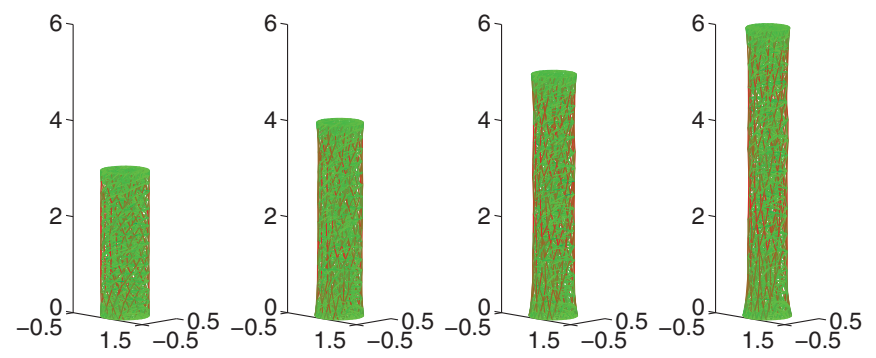

FIG. 10. (Color online) Resulting three-dimensional meshes for an increasingly deforming solid cylinder. Its initial dimensions are $D \times H=1 \times 3$, and the cylinder is retriangularized at each time step using a Delaunay algorithm [24]. The cylinder is subjected to an imposed height $h=H+0.5 t$. Snapshots correspond to times $t=0$, $t=2, t=4$, and $t=6$.

\section{B. Stress relaxation analysis}

We aim to test whether the one-dimensional strain evolution described in Sec. II C carries over to a network system. In the latter case, though, it is very likely that when the material is subjected to large deformations, cell rearrangements due to changes in the cell-cell connectivities will occur. In order to simulate these macroscopic topology changes, some authors have considered the presence of yield stresses in the constitutive law of the material [20]. We have here resorted to explicitly redefining the connectivity of the network by using a Delaunay triangularization, which has been applied with the help of the Qhull library [24]. We note that other discretizations and criteria to define the connectivity changes are possible, which may in turn affect the macroscopic stress-strain relationship. The analysis of these strategies is out of the scope of this work, but the interested reader may find a review, for instance, in Ref. [25].

We have analyzed the response of a two-dimensional and a three-dimensional network, forming, respectively, a 2D patch and a cylinder with a vertical axis. The 2D patch (see Fig. 7) is subjected to a linearly increasing displacement $x=X_{0}+0.5 t$, while the $3 \mathrm{D}$ cylinder has been stretched through the application of a prescribed height $h=H+0.5 t$. Figures 9 and 10 show the resulting deformed networks during the six initial time units of the simulation, where the elements

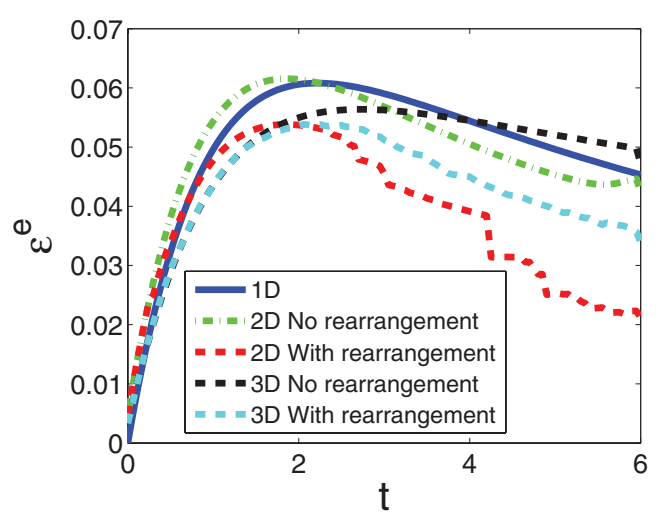

FIG. 11. (Color online) Stress evolution in a test with linearly increasing displacements for one element (1D), the 2D patch in Fig. 7, and a three-dimensional network that forms a vertical cylinder. The stresses in the 2D (3D) analysis have been computed by adding all the reactions at one end and dividing by the length (surface) at this end. Simulations with cell rearrangements indicate that the connectivity has been modified at each time step with a Delaunay triangularization. 


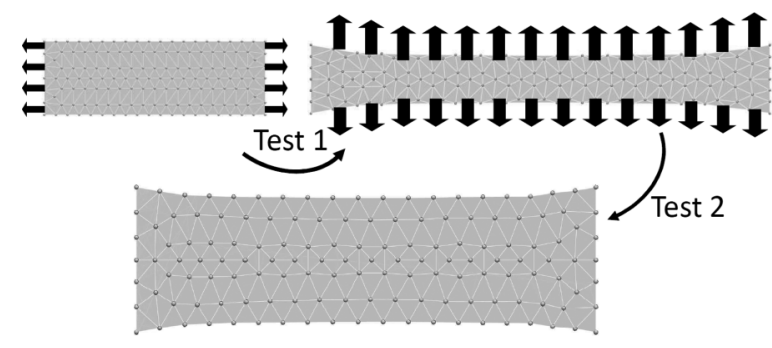

FIG. 12. Scheme of the two consecutive tests being analyzed: a horizontal displacement is applied on the right end of the 2D patch (Test 1), and a vertical displacement is applied on the top end of the patch after Test 1.

with higher positive elastic strains have been indicated in red (dark gray). The movies S1_movie and S2_movie in the Supplemental Material [26] also illustrate the deformed configurations and the Delaunay remeshing process.

The resulting stresses at one end of the domain measured as the sum of the horizontal (vertical) nodal forces per unit of length (surface) in the 2D (3D) network have been plotted in Fig. 11. For the two- and three-dimensional cases, simulations with and without cell rearrangements have been distinguished. In the former case, the network topology is modified at each time step, and the connectivity is triangularized with the Delaunay algorithm. It can be observed that the changes in the topology of the network also contribute to the softening process of the tissue. This is a numerical artifact, due to the presence of new elements that replace elements that had a larger strain energy. In fact, such numerical softening can be controlled by improving the approximated stress state of the new elements. In our model, we have averaged the stress state of the new elements from their nodal stress values. On the other hand, though, cells do rearrange, and do so with a consequent strain energy reduction. More accurate quantification of the energy cost of the cell rearrangement is needed in order to better account for the stress difference between deformations with constant and variable topology. Despite these differences, the trend of the curves in Fig. 11, and the instant of the maximum stresses coincide in the five cases considered here.



FIG. 13. (Color online) Evolution of the reactions for the two consecutive tests depicted in Fig. 12. The horizontal traction (Rx) is measured at the right boundary for Test 1 during the first 6 time units, while the vertical traction (Ry) is measured at the top boundary for Test 2 during the last 6 time units. The total test lasts 12 time units.
We have further analyzed the effects of the cell remodeling by applying an orthogonal strain after the two-dimensional test previously described. Figure 12 depicts the two sequential tests. After comparing the horizontal reaction per unit of length in Test $1, \mathrm{Rx}$, and the vertical reaction per unit of length during Test 2, Ry (see the plot in Fig. 13), we observe that the evolution of the reaction Ry without Delaunay is much lower than the values when using Delaunay triangularization. The difference may be explained by the fact that in the former case the trusses are in general very much horizontal after Test 1 , which renders them much softer in the orthogonal direction. When using Delaunay triangularization, no preferred direction is adopted, and a similar trend of the total reaction is measured in Test 1 and Test 2.

\section{DISCUSSION}

In this work we have proposed an evolution law of the macroscopic remodeling process which mimics the apparent measured viscosity. The proposed law in Eq. (1) aims to (i) reproduce the ability of the cell to adapt to the current strains and (ii) respond with a limited amount of sustained stress. Despite its simplicity, this evolution law is able to reproduce the viscoelastic response at small strains. The resting length changes have been combined with a purely linear elastic law, and the resulting active model has been compared against a linear Maxwell model. It has been shown that by using the following relation:

$$
\gamma \approx \frac{k}{\eta}
$$

the active model is equivalent to the Maxwell model, overall for the stress relaxation test and when applying oscillatory displacements. Although the responses of the two models are very similar, the active model provides a different physical interpretation of the resulting viscosity: the viscous response of the cell may be due to the resistance of the cell to remodel and not to the resistance of the fluid content to the strain rates.

The active model relays on the alternative expression of the cell rheological law given in (1) which explicitly includes controllable cell activity and whereby the viscous strain rate is replaced by the active lengthening. The model has been applied to tissues with large strains, but further experiments at this regime and more sophisticated rheology models are necessary in order to better match the observed nonlinear response of tissues such as cell stiffening [27] and cell softening [28]. The active element may be also combined with other rheological configurations in order to design more general active standard solid models, equivalent to the one depicted in Fig. 1(c).

So far, qualitative experimental evidence of the relationship between polymerization activity and tissue viscosity is reported in Refs. [11,29]. In the former reference, actin activity inhibition (smaller remodeling rate) results in a higher viscosity, similar to the equivalence between $\gamma$ and $\tau^{-1}$ in our results. In this work, we have suggested other quantitative analyses that may help to verify some of the outcomes of the model. For instance, the long-term evolution of the elastic strain (or stress) or the dependence of the maximum stress on the remodeling and (de-)polymerization rate shown in Fig. 4 could be experimentally analyzed. 
We note that despite the similarities between the Maxwell model and active element with $\gamma=\tau^{-1}$, the physical interpretation of the two models substantially differ. For instance, in the creep analysis with a linearly increasing applied load in a volume $V$, the viscous strain rates $\dot{\varepsilon}^{v}=\dot{\varepsilon}-\dot{\varepsilon}^{e}$ of the Maxwell model are associated to the dissipated power $P_{\text {dis }}=$ $\int_{V} \dot{\varepsilon}^{v} \sigma d v$. Instead, in the active element, the inelastic energy contributions correspond to the power spent by the cell to remodel. More specifically, disregarding passive dissipations powers, the energy balance of the active element reads

$$
P_{\mathrm{ext}}=P_{e}+P_{r}
$$

where the external power $\left(P_{\text {ext }}\right)$, elastic power $\left(P_{e}\right)$, and remodeling power $\left(P_{r}\right)$ contributions are given by

$$
P_{\mathrm{ext}}=\int_{V} \dot{l} f_{\mathrm{ext}} d v, \quad P_{e}=\int_{V} \dot{\varepsilon}^{e} \sigma d v, \quad P_{r}=\int_{V} \dot{L} Y d v .
$$

The quantity $Y$ corresponds to the necessary energy to stretch one unit the resting length of the filament. In a Maxwell model instead, the term $P_{r}$ must be replaced by the dissipated power $P_{\text {dis }}$. We note that if the active stresses are also accounted for, as in the case of the models in Refs. $[4,8,18]$, this additional energy source should be incorporated in the left-hand side of Eq. (7).

The same basic ideas can be extended to continua. In this case, though, the definition of multiple parameters $\gamma$ for each direction is required. In the present implementation, we have resorted to a $1 \mathrm{D}$ rheology model, which allows us to obtain the anisotropic response as a result of the different orientations of the elements. Moreover, the development of the nondiscrete spectrum may be considered by resorting to unidimensional linear superposition of Boltzmann and the use of the general creep function and relaxation functions [1].

We expect to further extend the model to nonlinear elastic constitutive laws that may include stiffening [2] and softening [28] phenomena and also quantify more accurately the relation between cell viscoelasticity and intracellular remodeling and intercellular topology changes.

We also note that we have not simulated the chemical signaling that triggers the remodeling of the cytoskeleton but just the observed macroscopic deformations that represent such remodeling process. Models where the dynamics of biopolymers and the cytoskeleton are explicitly represented have been published elsewhere [30-33]. Notwithstanding the simplified representation of our model, we have been able to simulate cell-cell interactions and the global response of tissues by extending the essential ideas of the unidimensional model. We have showed that the simulated multidimensional cellular networks inherit the properties of the unidimensional formulation, such as the relative evolution of the stresses or the frequency dependence of the phase angle.

The role of cell activity and its effects on cell rheology has been well reported in the literature. These models contain two main ingredients: changes in the cell reference (stress free) configuration and additional stresses due to cell activity. Examples of the first case are the seminal model of Huxley [34], where relative sliding of actin and myosin are considered, or the work in Ref. [35] where the material is able to flow to a different well in the energy landscape due to the rearrangement of drops. In the context of embryogenesis, stress-dependent deformations of the reference configuration are considered, for instance, in Refs. [36,37], following the ideas of Beloussov's restoration hypotehesis [38]. Examples of the second case may be found, for instance, in Refs. [3,4,8,16-18]. Our present model could be included in the first group. However, we have additionally analyzed the effects on the cell rheology that the changes in the reference configuration may induce. We have shown that, for the particular form employed here and in the small strain regime, the two phenomena are closely related.

The resulting picture of the proposed model is in fact closer to a solid with active changes than a passive viscoelastic material, as it is often used in numerical simulations. Consequently, we are more inclined to relate the cell viscosity to the cell activity and its remodeling capability rather than to its fluid content, whose viscous properties are orders of magnitude lower than those of the cellular tissue.

\section{ACKNOWLEDGMENTS}

The authors are grateful to Xavier Trepat and Vito Conte at the Institute for Bioengineering of Catalonia (Barcelona, Spain) for their fruitful and helpful discussions.
[1] Y. C. Fung, Biomechanics: Mechanical Properties of Living Tissues, 2nd ed. (Springer, New York, 1993).

[2] D. Mizuno, C. Tardin, C. F. Schmidt, and F. C. MacKintosh, Science 315, 370 (2007).

[3] F. C. MacKintosh and A. J. Levine, Phys. Rev. Lett. 100, 018104 (2008).

[4] A. Besser, J. Colombelli, E. H. K. Stelzer, and U. S. Schwarz, Phys. Rev. E 83, 051902 (2011).

[5] T. Gregor, W. Bialek, R. R. de Ruyter van Steveninck, D. W. Tank, and E. F. Wieschaus, Proc. Natl. Acad. Sci. USA 102, 18403 (2005).

[6] T. Bittig, O. Wartlick, A. Kicheva, M. González-Gaitán, and F. Jülicher, New J. Phys. 10, 063001 (2008).

[7] G. Forgacs, R. A. Foty, Y. Shafrir, and M. S. Steinberg, Biophys. J. 74, 2227 (1998).
[8] K. Kruse and F. Jülicher, Phys. Rev. E 67, 051913 (2003).

[9] D. Gonzalez-Rodriguez, K. Guevorkian, S. Douezan, and F. Brochard-Wyart, Science 338, 910 (2012).

[10] K. Luby-Phelps, Int. Rev. Cytol. 192, 189 (2000).

[11] D. Azevedo, M. Antunes, S. Prag, X. Ma, U. Hacker, G. W. Brodland, M. S. Hutson, J. Solon, and A. Jacinto, PLoS ONE 6, e23964 (2011).

[12] B. Fabry, G. N. Maksym, J. P. Butler, M. Glogauer, D. Navajas, and J. J. Fredberg, Phys. Rev. Lett. 87, 148102 (2001).

[13] P. S. Janmey, S. Hivdt, J. Käs, D. Lerche, A. Maggs, E. Sackmann, M. Schliwa, and T. P. Stossel, J. Biol. Chem. 269, 32503 (1994).

[14] F. C. MacKintosh, J. Käs, and P. A. Janmey, Phys. Rev. Lett. 75, 4425 (1995)

[15] F. Gittes and F. C. MacKintosh, Phys. Rev. E 58, R1241 (1998). 
[16] K. I. Morozov and L. M. Pismen, Phys. Rev. E 81, 061922 (2010).

[17] T. B. Liverpool, M. C. Marchetti, J. F. Joanny, and J. Prost, Europhys. Lett. 85, 18007 (2009).

[18] A. Besser and U. S. Schwarz, New J. Phys. 9, 425 (2007).

[19] W. N. Findley, K. Onaran, and W. J. Lai, Creep and Relaxation of Nonlinear Viscoelastic Materials: With an Introduction to Linear Viscoelasticity (Dover, London, 1989).

[20] D. Ambrosi and L. Preziosi, Biomech. Model. Mechanobiol. 8, 397 (2009).

[21] M. de Berg and O. Cheong, M. van Kreveld, and M. Overmars, Computational Geometry. Algorithms and Applications, 3rd ed. (Springer-Verlag, Heidelberg, 2008).

[22] J. J. Muñoz, V. Conte, N. Asadipour, and M. Miodownik, Mech. Res. Comm. 49, 44 (2013).

[23] M. Hahn, T. Wallmersperger, and B.-H. Kröplin, Comput. Mater. Sci. 50, 391 (2010).

[24] C. B. Barber, D. P. Dobkin, and H. T. Huhdanpaa, ACM Trans. Math. Soft. 22, 469 (1996). http://www.qhull.org.

[25] P. Pathmanathan, J. Cooper, A. Fletcher, G. Mirams, P. Murray, J. Osborne, J. Pitt-Francis, A. Walter, and S. J. Chapman, Phys. Biol. 6, 036001 (2009).

[26] See Supplemental Material at http://link.aps.org/supplemental/ 10.1103/PhysRevE.88.012708 for Movies with deformed twoand three-dimensional networks.
[27] C. Storm, J. J. Pastore, F. C. MacKintosh, T. C. Lubensky, and P. A. Janmey, Nature 435, 191 (2005).

[28] X. Trepat, L. Deng, S. S. An, D. Navajas, D. J. Tschumperlin, W. T. Gerthoffer, J. P. Butler, and J. J. Fredberg, Nature 447, 592 (2007).

[29] X. Ma, H. E. Lynch, P. C. Scully, and M. S. Hutson, Phys. Biol. 6, 036004 (2009).

[30] C. J. Cyron and W. A. Wall, Phys. Rev. E 82, 066705 (2010).

[31] T. Kim, W. Hwang, H. Lee, and R. D. Kamm, PLOS Comp. Biol. 5, e10000439 (2009).

[32] C. Tamulonis, M. Postmea, H. Q. Marlow, C. R. Magie, J. de Jong, and J. Kaandorp, Dev. Biol. 351, 217 (2011).

[33] P. Van Liedekerke, E. Tijskens, and H. Ramon, Phys. Rev. E 81, 061906 (2010).

[34] A. F. Huxley, Prog. Biophys. Biophys. Chem. 7, 255 (1957).

[35] P. Sollich, F. Lequeux, P. Hébraud, and M. E. Cates, Phys. Rev. Lett. 78, 2020 (1997).

[36] J. J. Muñoz, V. Conte, and M. Miodownik, Biomech. Model. Mechanobiol. 9, 451 (2010).

[37] L. A. Taber, Biomech. Model. Mechanobiol. 7, 427 (2008).

[38] L. V. Beloussov, S. V. Saveliev, I. I. Naumidi, and V. V. Novoselov, Int. Rev. Cytol. 150, 1 (1994). 